\title{
RADICAL, DISRUPTIVE, DISCONTINUOUS AND BREAKTHROUGH INNOVATION: MORE OR THE SAME?
}

\author{
ADRIAN KOVACS \\ KU Leuven, Department of Management, Strategy and Innovation (MSI), Faculty of Business and \\ Economics (FEB), B-3000 Leuven, Belgium \\ CRISTINA MARULLO \\ Sant'Anna School of Advanced Studies, Pisa (IT)- Institute of Management \\ DENNIS VERHOEVEN \\ KU Leuven, Department of Management, Strategy and Innovation (MSI) \\ BART VAN LOOY \\ KU Leuven, Department of Management, Strategy and Innovation (MSI)
}

\section{INTRODUCTION}

Within the field of innovation and technology management there is consensus that not all innovations are alike. While a vast majority of innovations imply incremental improvements and/or display a modest impact, a (much smaller) number of innovations are considered as 'exceptional', since they significantly depart from previous practices and/or have a more profound impact (see for instance Schumpeter, 1934; Baumol, 2004; Ahuja \& Lampert, 2001; Bower \& Christensen, 1995; O'Connor \& Rice, 2001). Within the literature, different constructs have been advanced to denote 'exceptional' innovations whereby the labels 'radical' (Chandy \& Tellis, 1998; Ettlie, Bridges, \& O'Keefe, 1984), 'breakthrough' (Barnholt, 1997; Mascitelli, 2000), 'discontinuous' (Danneels, Kleinschmidt, \& Cooper, 2001; McKee, 1992) and 'disruptive' (Christensen, Verlinden, \& Westerman, 2002; Markides \& Anderson, 2006) figure most prominently. In the past, concerns have been uttered that the simultaneous use of these constructs might result in ambiguity regarding the interpretation of the underlying empirical phenomena, especially when such labels are defined loosely and/or are being used interchangeably while referring to different phenomena and processes (Linton, 2009). This ambiguity is suspected to have negative consequences, with some scholars going as far as to assert that the resulting confusion might hamper both theory development and the distilment of insights and advices relevant and useful for practitioners (Garcia \& Calantone, 2002;

Gatignon, Tushman, Smith, \& Anderson, 2002).

In this paper, we explicitly revisit these concerns by engaging in a systematic, bibliometric review of a comprehensive sample of publications as they appeared in the Web of Science (WOS) covered journals during the last three decades (1985-2016). To assess the distinctiveness (or lack thereof) of the labels in use, we analyse articles that explicitly refer to either 'radical', 'breakthrough', 'discontinuous' and 'disruptive' innovation and that appeared in the domains of business, management and economics, both in terms of their theoretical foundations as well as in terms of their thematic orientation. To this purpose, we rely on cocitation analysis and bibliographic coupling, two similarity measures used to study the relatedness between documents based on (dis-) similarities in referencing/citing patterns. We complement the bibliometric analysis with a content analysis of the top-hundred most-cited publications in our dataset in order to develop a more thorough understanding of the meaning of and relations between the different definitions and operationalisations on the labels of interest. The co-citation 
analysis reveals that the constructs 'radical', 'breakthrough' 'discontinuous' and 'disruptive' innovation rely to a large extent on similar 'foundations': for articles that receive at least 10 citations, the amount of shared references ranges between 51 and $90 \%$. The analysis based on bibliographic coupling signals five distinct clusters of thematically related publications, but also here, no exclusive relationship between themes and labels is to be observed: rather than representing silos of publications that are developed under a specific label (e.g. 'radical', 'breakthrough', 'discontinuous' or 'disruptive'), these clusters reflect themes commonly pursued across labels. At the same time, these publications display aggregated growth rates above average, with radical innovation starting to account for the 'lion share' of publications, especially during the last decade. As such, these observations might suggest that it is only a matter of time, before the field converges towards a 'dominant term'. This is however not confirmed when complementing the bibliometric analysis with a content analysis of the definitions advanced in the most-cited publications $(n=100)$. Here it becomes apparent that two conceptually distinctive dimensions are being used to characterize the nature of exceptional innovations: (i) novelty and (ii) impact. While the resulting quadrant could host four distinctive terms, we currently do not observe a situation where the majority of papers related to one label appears in only one quadrant. And while this does not seem to jeopardize the growth of scholarship, we do conclude by arguing that our understanding - of the complex, underlying phenomena - would benefit from more rigor and accuracy both in terms of defining and in terms of measuring the processes under study.

\section{DATA AND METHODS}

We relied on the 'topic search option' in the WOS database to retrieve publications that coin the term 'innovation' and any of the terms 'radical', 'breakthrough', 'discontinuous' and/or 'disruptive' in either the title, keywords or abstract fields. In accordance with our objectives, we limited our search to publications in the WOS subject-categories 'Management', 'Economics', 'Business' and/or 'Operations Management and Research Methods' and to the document types 'Article', 'Editorial', 'Note', 'Letter' and 'Review'. All publications containing the relevant keywords starting from January 1985 up to November 2016 were added to the initial dataset. Our search key yielded 2,347 publications for which we downloaded the bibliographical records. In a first step, we identified and removed from our dataset 118 publications that had no cited references in common with any of the other publications. In a second step, we identified the 10-percentile of publications that had lowest number of cited references in common with other publications. Three researchers performed a manual check (title, abstract and keywords fields) independently to identify and remove irrelevant articles. The final dataset for further analysis consisted of 2.081 publications. These publications cite 65,284 publications and are cited by 36,117 publications.

We applied two bibliometric techniques - bibliographic coupling and co-citation analysis to analyse the thematic relatedness between publications. The fundamental assumption underlying these techniques is that the degree of overlap between the cited references of a focal pair of publications is representative of the thematic relatedness between these publications. The main difference between bibliographic coupling and co-citation analysis lies in the locus of citation patterns. Bibliographic coupling is a technique based on an analysis of the references these publications cite, whereas co-citation analysis is a technique based on an analysis of citations received. To determine the relatedness between publications we calculated the association strength measure for each pair of focal publications (Van Eck and Waltman, 2009). The association strength measures the co-occurrence frequency of citations made (in bibliographic coupling) or citations received (in co-citation analysis) considering the total number of linkages of each focal publication 
as well as the total number of linkages within the entire set of focal publications:

$$
A S_{i j}=\frac{C_{i j}}{C_{i} C_{j}}
$$

Whereby $C i j$ denotes the number of linkages (i.e. references/citations) shared by publications $i$ and $j, C_{i}$ denotes the total number of linkages of publication $i, C_{j}$ denotes the total number of linkages of publication $j$. In bibliographic coupling the linkages denote citations given whereas in co-citation analysis they denote citations received.

\section{RESULTS}

We address the following two questions: (1) to what extent do publications using different labels overlap with respect to the scholarly works they cite (i.e. are they distinct in terms of origins/ theoretical foundations?) and (2) to what extent do these publications overlap in the topics that they address (i.e. have they common or different thematic orientations?) We rely on co-citation analysis to address the first question and bibliographic coupling to address the second question.

\section{The Origins of the Scholarship on 'Exceptional' Innovation}

To quantify co-citations among publications advancing different labels, we calculated a Jaccard-index measuring the overlap among references cited at least 10 times by our sample of publications (see Table 1). This measure calculates the number of normalized co-citations among two sets of publications referring to different labels as a share of potential co-citations, with a greater value indicating a higher overlap between the two sets.

Table 1 about here

The Jaccard-Index scores highlight that a significant share of the 1,326 references that are cited at least 10 times are co-cited by publications representing different labels. They indicate that at least $50 \%$ of the cited references of any pair of labels overlap, with the overlap being the highest for pairs involving the set of publications referring to multiple labels. Jointly, the results based on co-citation analysis signal a significant overlap with respect to the scholarly works that publications referring to different labels rely upon.

\section{The Thematic Orientation of the Scholarship on 'Exceptional' Innovation}

To visualize the overlap in citation patterns for each pair among our set of 2,081 publications, we mapped the network of publications based on bibliographic coupling using the association strength measure. In Map 1 we present the bibliographic network enabling the identification of clusters of thematically related publications. In this figure the nodes (publications) are described by the labels themselves (e.g. RAD for 'radical'). The size of each node corresponds with the number of citations received and the distance between a pair of nodes corresponds to the overlap in their citation patterns, with a shorter distance corresponding with a greater overlap. The 
location of a node near the centre of the map indicates that the underlying publications' citation pattern greatly overlaps with that of many other publications. Finally, the colours (red, green, blue, yellow and purple respectively) represent thematic clusters. The classification of a publication into a cluster indicates that its citation pattern has a greater overlap with other publications within that cluster than with publications that are classified into other clusters.

Map 1 about here

We observe that the bibliographic network of research on 'exceptional' innovation is relatively connected, since most publications have cited references that are in common with many other publications. Also, the proximity of different labels indicates that pairs of focal publications using different labels often have a greater overlap in references than pairs of focal publications referring to the same label. From these results we derive that, in the body of literature examined, similar labels seem to be addressing different topics and, at the same time, different labels are used to denote similar topics. In other words, both publications representing the same and those representing different labels can be thematically related. To further shed light on the thematic orientation of the literature on exceptional innovation, we identify the main topics that each of the five clusters represent and further assess the ways in which the top-publications within these clusters define and measure the phenomena of interest (Map 2). The content analysis reveals that the different labels are neither being defined consistently nor in an exclusive manner. Whereas definitions do rely on two distinctive underlying dimensions - namely novelty and impact, the current body of literature does not allow for a comprehensive categorization reflecting four clearly distinctive quadrants.

Map 2 about here

\section{DISCUSSION AND CONCLUSION}

Despite concerns expressed previously, most notably by Garcia \& Calantone (2002) and Gatignon et. al. (2002), scholarship on exceptional innovation has experienced considerable growth during the past 15 years. This growth is mostly accounted for by the increasing number of publications referring to the label 'radical'. Although labels are seldom used jointly within one publication, authors having multiple publications often refer to different labels in different publications. The co-citation analysis of the references cited most by scholarship referring to a certain label signals considerable overlap with respect to the core academic contributions that these scholarships build upon. From this analysis we can infer that the 'intellectual' origins of different labels are highly related, which in turn might explain their interchangeable use in scholarship on exceptional innovation. In terms of thematic orientation, bibliographic coupling reveals five, overlapping, clusters; none of them adhering exclusively to one specific label. Combined these observations seem to suggest convergence, which might eventually result in one 'dominant term'.

The label 'radical' innovation seems to be in the lead position in this respect, as the term starts to account for the lion share of publications. Such a contention was confirmed by a content analysis of the definitions and delineations used by authors adhering to the terms under study. Two 
observations stand out. First, a significant number of contributions is rather implicit in terms of delineating or defining the exact nature of the terms advanced. In total, 44 of the most cited papers do not provide an explicit definition of the 'central construct'. Second, the papers that do advance an explicit delineation of the type of innovation under study tend to rely on two, distinct, dimensions: (1) novelty and (2) impact. Novelty is defined as reflecting the obviousness of an innovation compared to the state-of-the art at the moment of creation, whereas impact refers to effects that unfold over time. Stated otherwise, novelty can be assessed as soon as an innovation is being conceived, whereas the assessment of impact implies a process that might span a considerable time period. As our analysis clarifies, there is no consensus about the dimensions that focal labels should encompass. Whilst every focal label has a defining underlying dimension either novelty or impact - that is universally recognized in existing scholarship, there is no consensus with regards to whether a focal label also encompasses the other dimension. The labels 'radical' and 'discontinuous' tend to refer to innovations that are highly novel whilst the labels 'breakthrough' and 'disruptive' tend to signal innovations that have a high impact. However, for some scholars the labels 'radical' and 'discontinuous' imply innovations that are highly novel, regardless of their potential impact, whereas for others these labels equate to innovations that are both novel and have a high impact. In a similar vein, some scholars use the labels 'breakthrough' and 'disruptive' to describe innovations that have a high impact, regardless of whether they are novel, whilst others use these labels to refer to innovations that both have a high impact and are highly novel.

This is clearly not a desired state of affairs. Whereas the use of different labels per se, might not be so problematic, clarity on the level of underlying dimensions seems highly desirable. A first reason resides in avoiding Type I and/or Type II errors. By confounding both dimensions, one might convey the message that impactful innovations will always be novel and vice versa. A second reason resides in the nature of the phenomena including their antecedents. If one is concerned with novelty (and the creation thereof), knowledge creation processes come to the forefront (including micro-foundations in terms of cognition and creativity) whereas impact centres around diffusion dynamics, including the interplay with existing resources, systems and broader socio-technical systems. Both dimensions introduce a temporal element, albeit in a different manner: novelty can almost be conceived as an 'ex-ante' dimension of exceptional innovation whereas impact can only be assessed ex-post (and this assessment may vary over time). Especially this 'temporal' distinction between both dimensions could inspire scholars to expostulate innovation trajectories rather than innovation types when developing insightful classifications and typologies.

\section{REFERENCES AVAILABLE FROM THE AUTHORS}

\section{ACKNOWLEDGEMENTS}

This study is part of the research work conducted with Alberto Di Minin, Andrea Piccaluga (Sant'Anna School of Advanced Studies) and Dries Faems (WHU) within the EIBURS Lines of Research 2015-2018. The authors are grateful to the European Investment Bank (EIB) for funding and support and to Mark Pevsner for his insightful comments. While the authors acknowledge their contribution, the content of this manuscript does not necessarily reflect the position of the EIB. 
Table 1. Overlaps Among References Cited at Least 10 Times (Jaccard-Index Scores)

\begin{tabular}{l|rrrrr} 
& RAD_ONLY & DRU_ONLY & CON_ONLY & BRE_ONLY & MULTI \\
\hline RAD_ONLY & $\mathrm{X}$ & 0,71 & 0,86 & 0,63 & 0,90 \\
DRU_ONLY & & $\mathrm{X}$ & 0,67 & 0,51 & 0,67 \\
CON_ONLY & & & $\mathrm{X}$ & 0,59 & 0,80 \\
BRE_ONLY & & & & $\mathrm{X}$ & 0,60 \\
MULTI & & & & & $\mathrm{X}$
\end{tabular}

Map 1. A Visualization of Clusters of Thematically Related Publications Referring to different labels

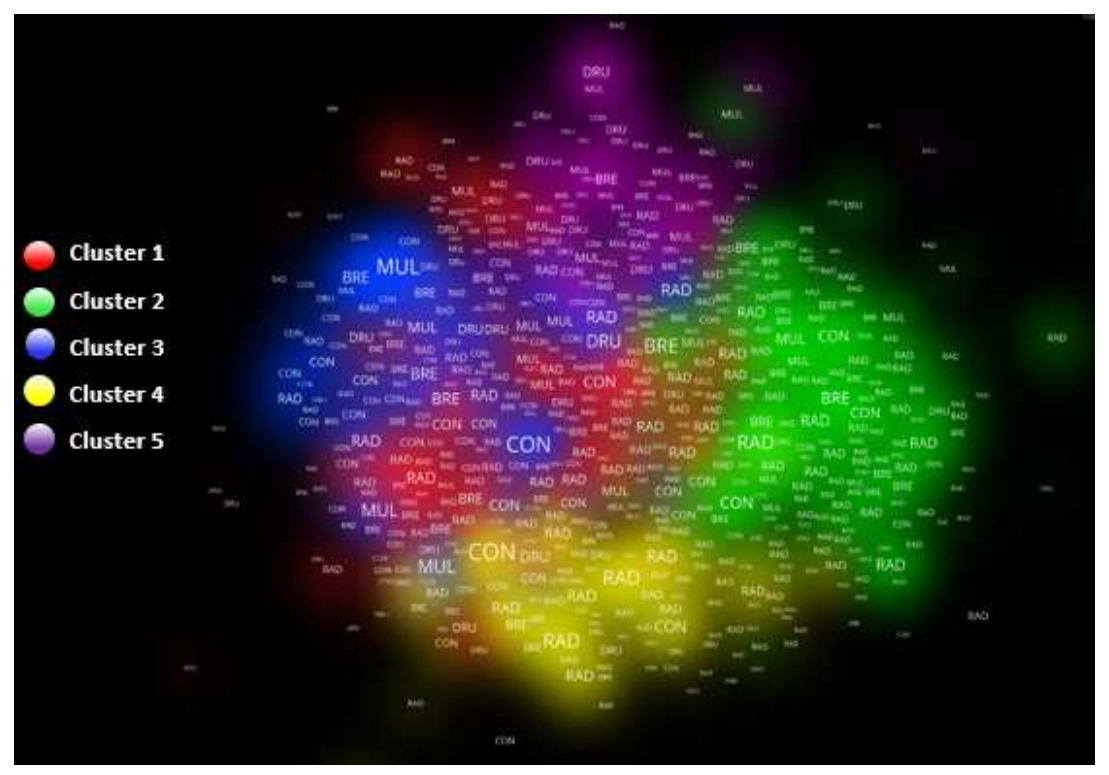

Map 2. A Visualization of Clusters of Thematically Related Publications Displaying First Author and Publication Year

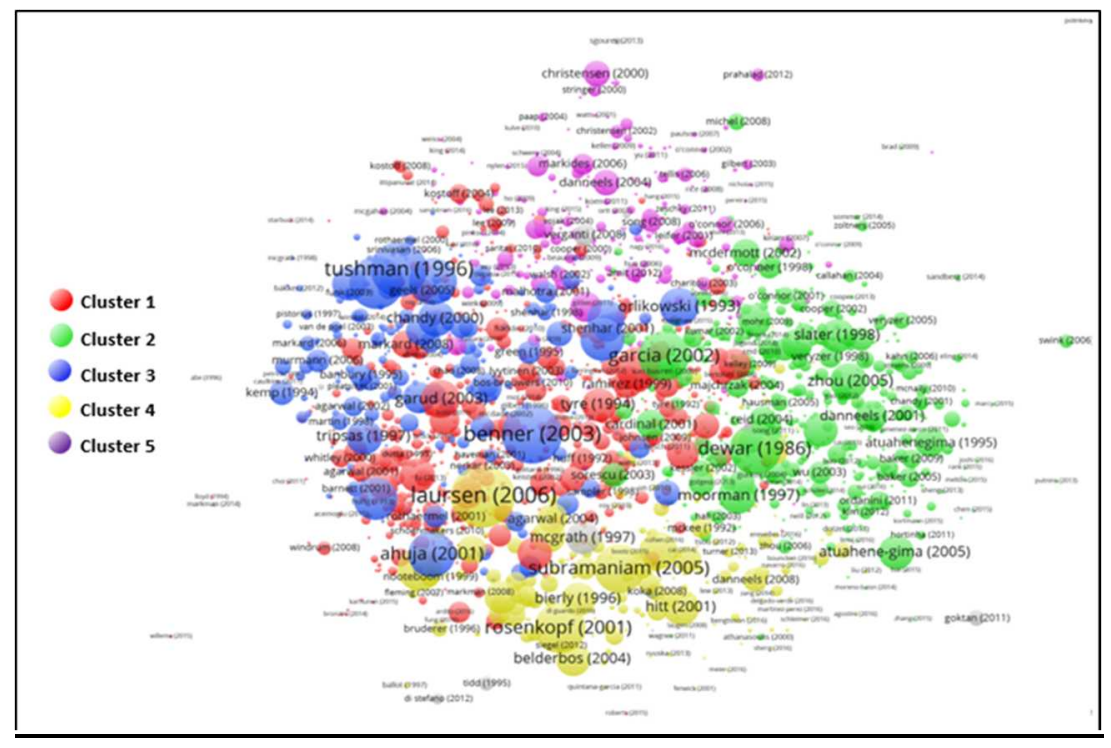

